\title{
Method for Thermal Pain Level Prediction with Eye Motion using SVM
}

\author{
Kohei Arai \\ Department of Information Science \\ Saga University \\ Saga City, Japan
}

\begin{abstract}
Method for thermal pain level prediction with eye motion using SVM is proposed. Through experiments, it is found that thermal pain level is much sensitive to the change rate of pupil size rather than pupil size itself. Also, it is found that the number of blinks shows better classification performance than the other features. Furthermore, the eye size is not a good indicator for thermal pain. Moreover, it is also found that user respond to the thermal stimulus so quickly ( 0 to 3 sec.) while the thermal pain is remaining for a while $(4$ to $17 \mathrm{sec}$.) after the thermal stimulus is removed.
\end{abstract}

Keywords-Eye motion; thermal pain; support vector machine; thermal stimulus; classification

\section{INTRODUCTION}

The locus coeruleus (LC) signals salience to sensory stimuli and these responses can modulate the experience of pain stimuli. The pupil dilation response (PDR) to noxious stimuli is thought to be a surrogate for LC responses, but PDR response to Peltier-controlled noxious heat stimuli, the most commonly used method in experimental pain research, has not been described [1].

A bedridden person is a person who has become a plant state, a person who cannot speak words well. Such people are difficult to convey their feelings. Currently, researches on gaze input, etc. are being conducted as a way for people who feel difficult to convey feelings to emotion. However, there are times when I have something I want to convey without having time to spare time of line of sight. Therefore, there is a strong demand to create methods and systems which allow pain level prediction and representation for disable persons.

There are not so small number of methods of thermal pain level prediction, such as MEG, MRI, fMRI, EOG, EEG, etc. These, however, are not so easy to measure and prediction of thermal pain due to the fact that it is difficult to recognize thermal pain with measured data and also it does cost for the measuring instruments. Also, these are not accurate enough (around $75 \%$ of accuracy with some time delay) [1].

Pupillary responses to thermal pain stimulation in healthy volunteers (Lauren A. Banker) ${ }^{1}$. Pupil responses and pain ratings to heat stimuli: Reliability and effects of expectations and a conditioning pain stimulus (James C. Eisenach et al.) [1]. Although these studies are based on only the pupillary response, in this research I add elements of other eye motion. Towards a physiology-based measure of pain: patterns of human brain activity distinguish painful from non-painful thermal stimulation (Justin E. Brown et al. ${ }^{2}$ ) [2]. Although this study is a study of brain activity by fMRI data using classification method of Support Vector Machine: SVM, this research is a study using eye motion data using SVM.

With regard to the psychological status monitoring with eye motion, rescue system for elderly and disabled persons using wearable physical and psychological monitoring system is proposed [3]. Also, a method for psychological status estimation by gaze location monitoring using Eye-Based Human-Computer Interaction: EBHCI is proposed and validated [4]. On the other hand, a method for psychological status monitoring with line of sight vector changes (Human eyes movements) detected with wearing glass is developed [5].

Frequent physical health monitoring as vital sign with psychological status monitoring for search and rescue of handicapped, disabled and elderly persons is developed [6]. Meanwhile, psychological status monitoring with cerebral blood flow: CBF, Electroencephalogram: EEG and ElectroOculogram: EOG measurements is proposed and validated [7]. Relations between psychological status and eye movements are investigated [8] together with psychological status monitoring with cerebral blood flow, electroencephalogram and electrooculogram measurements [9]. Then, rescue system with sensor network for physical and psychological health monitoring is summarized [10].

In this paper, a new approach for thermal pain level prediction is proposed. Namely, a method for thermal pain level prediction with human eye motions, in particular, with pupil size change rate using SVM is proposed. There are previously proposed methods which use pupil size [11]. It is found that thermal pain level is much sensitive to pupil size change rate than pupil size. Such this sensitivity analysis is conducted.

The next section describes the proposed method for prediction of thermal pain level followed by experimental method and results. Then, the results are discussed followed by conclusion. Finally, future research work is described.

\footnotetext{
${ }^{1}$ https://steinhardt.nyu.edu/appsych/psych_cafe
}

\footnotetext{
${ }^{2}$ http://journals.plos.org/plosone/article?id=10.1371/journal.pone.0024124
} 


\section{METHOD FOR THERMAL PAIN PREDICTION WITH EYE MOTION USING SVM}

\section{A. System Configuration}

Near Infrared: NIR camera which is mounted on a glass is used for acquisition of eye images. Fig. 1 shows the outlook of the proposed system.

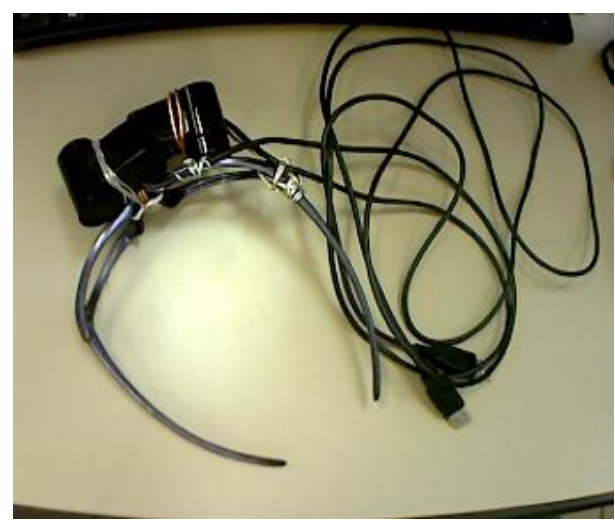

Fig. 1. Outlook of the system configuration.

NIR camera imagery data is acquired through USB interface to PC. The outlook of the camera is shown in Fig. 2 while major specification of the camera is shown in Table I.

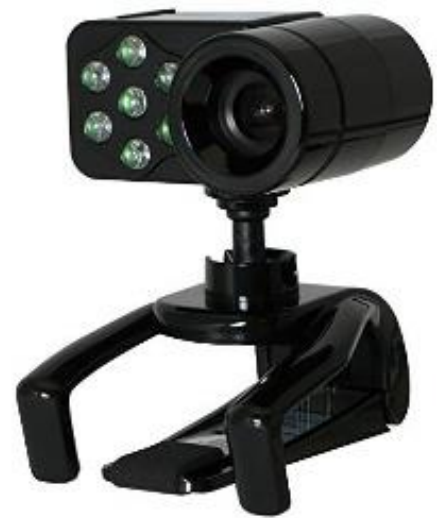

Fig. 2. Outlook of the NIR camera used.

TABLE I. MAJOR SPECIFICATION OF THE NIR CAMERA USED

\begin{tabular}{|l|l|}
\hline Weight & $200 \mathrm{~g}$ \\
\hline Size (dimension) & $21.8 \times 14.8 \times 7.6 \mathrm{~cm}$ \\
\hline Camera Name & DC-NCR13U \\
\hline
\end{tabular}

\section{B. Thermal Pain Level Prediction Method}

From the acquired eye image 1) eye size, eyelid width; 2) pupil size; 3 ) the number of blink for a time interval (blink frequency); 4) pupil size change rate; 5) pupil center location changes; and 6) pupil center location change rate are selected as feature components for thermal pain level prediction. Using relations between these features and subjective evaluation results of thermal pain, thermal pain is evaluated with the features through regressive analysis.
C. SVM

SVM is well known classification method which allows classify the data into the previously designated class categories. In the proposed method, two class categories, pain and not pain are designated. By using the feature components, SVM based classification is applied.

\section{EXPERIMENTS}

\section{A. Experimental Configuration}

Fig. 3 shows the experimental configuration which consists of the hot water pot with temperature measuring instrument and NIR camera mounted glass.

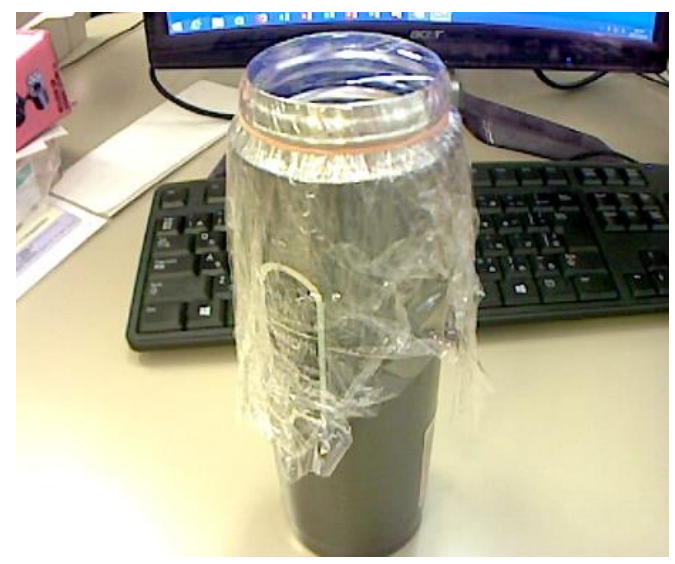

Fig. 3. Experimental configuration.

\section{B. Experimental Configuration}

Without any thermal stimulus, 50 seconds of eye images are acquired with 30 frame/second. After that, 10 seconds of eye images are acquired with 40 degree Celsius of the pot attached to the user's arm. These processes are repeated for six times, 600 seconds of eye images are acquired in total as shown in Fig. 4.

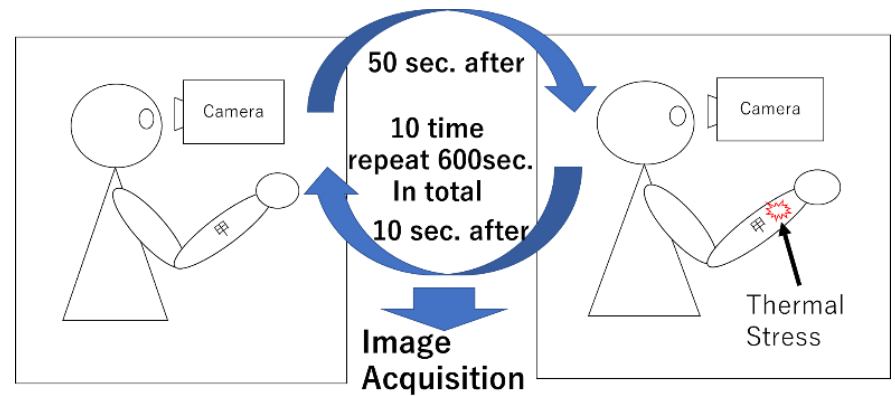

Fig. 4. Procedure of the experiment

\section{Preliminary Results}

Fig. 5(a) shows eye images when the user is having thermal stimulus while Fig. 5(b) shows those as the user is not having any thermal stimulus. It is quite obvious that pupil size as for having thermal stimulus is larger than that for having no thermal pain. Also, Fig. 5(c) shows eye image when the user is blinking. 

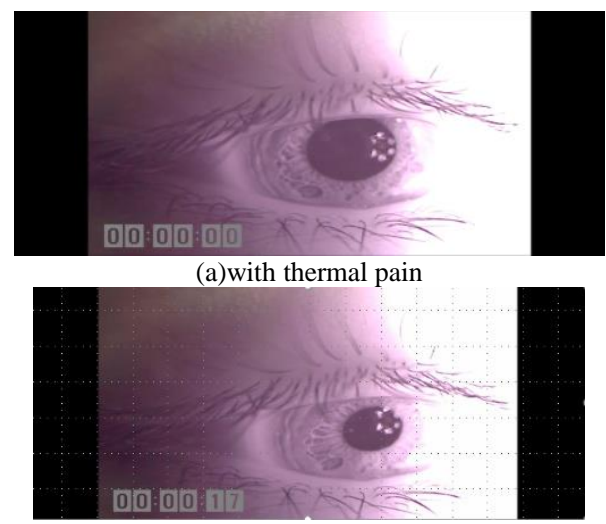

(b)without thermal pain

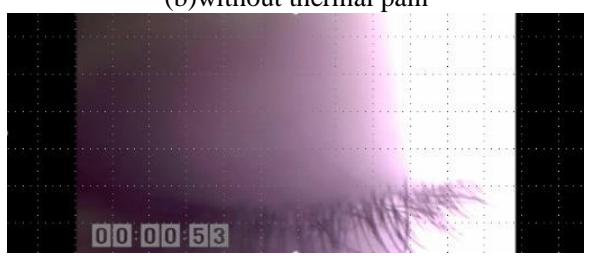

(c)Blinking

Fig. 5. Examples of eye images when user is having thermal pain and no thermal pain as well as blinking.

\section{Pupil Size}

Fig. 6(a) shows relation between thermal stimulus and pupil size. Meanwhile, Fig. 6(b) shows the result from linear regressive analysis between thermal stimulus and pupil size.

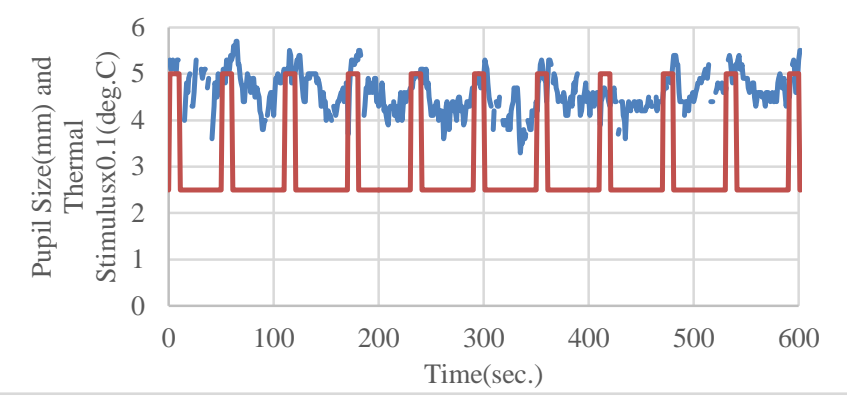

(a) Relation

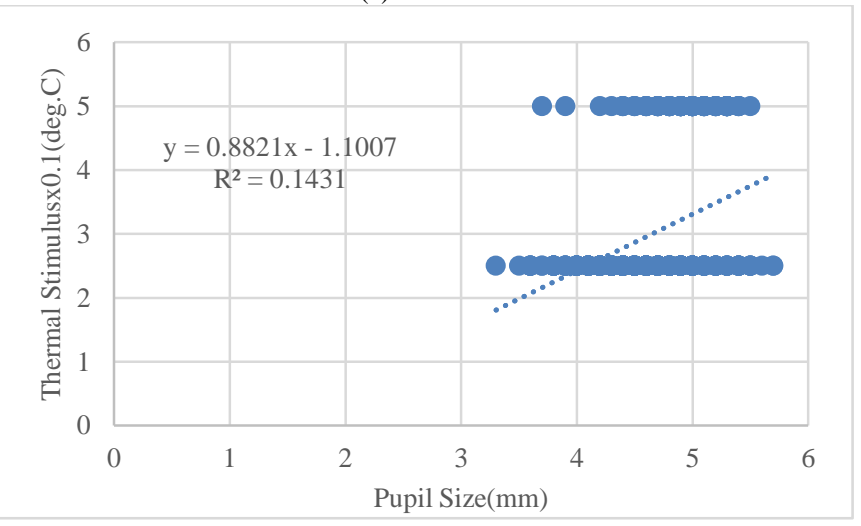

(b) Regressive analysis

Fig. 6. Relation between thermal stimulus and pupil size.

Although there is definite relation between thermal stimulus and pupil size, correlation coefficient between both is not so high, around 0.378 . On the other hand, response time of pupil size changes against thermal stimulus is shown in Fig. 7. Leading edges of the response are so quick in comparison to the trading edges. Namely, user respond to the thermal stimulus so quickly ( 0 to $3 \mathrm{sec}$.) while the thermal pain is remaining for a while (4 to $17 \mathrm{sec}$.) after the thermal stimulus is removed.

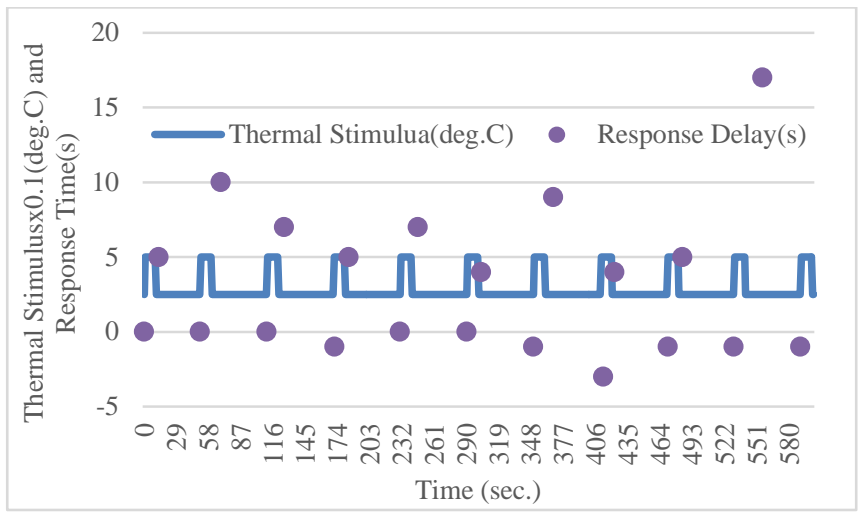

Fig. 7. Response time of pupil size changes against thermal stimulus.

\section{E. Eye Size}

Eye size is defined as the distance between the upper and lower eyelids. Usually, human eye size is getting large when users are surprising. Therefore, it is considered that eye size is one of the indicators for thermal pain. Fig. 8(a) shows the time series of eye size data against thermal stimulus while Fig. 8(b) shows the relation between both.

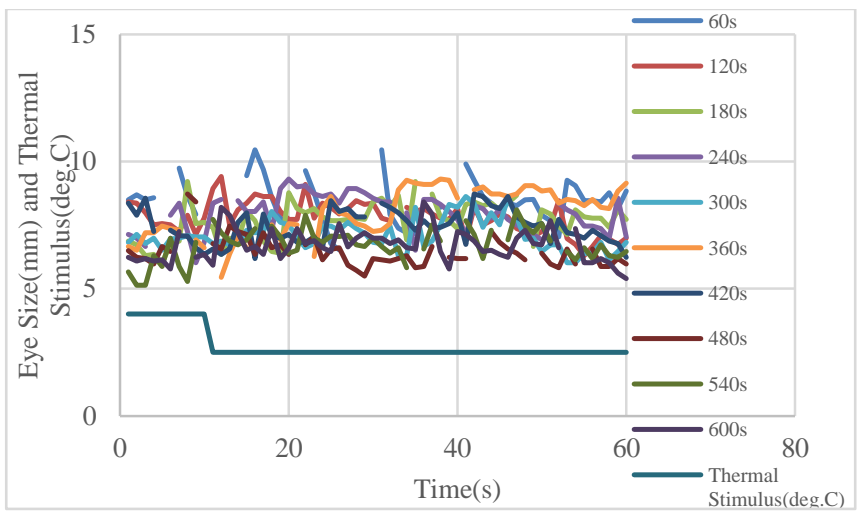

(a) Time series

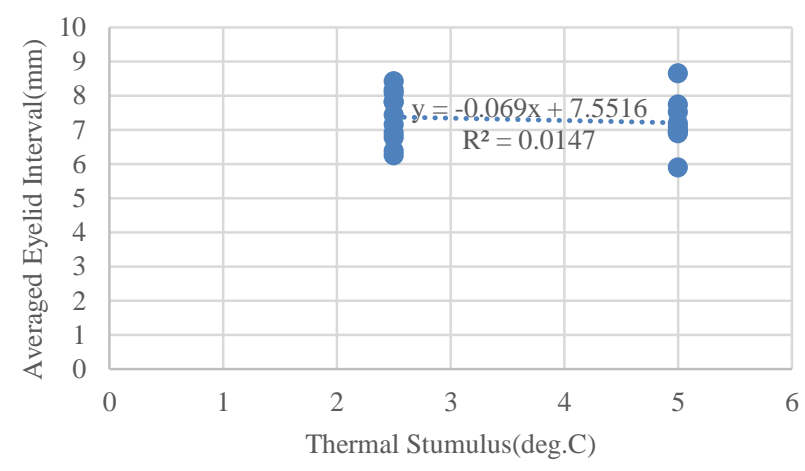

(b) Relation

Fig. 8. Relation between eye size and thermal stimulus. 
As shown in Fig. 8, relation between eye size and thermal stimulus is not high (correlation coefficient is around 0.1212). Therefore, eye size is not changed so much for thermal stimulus and no thermal stimulus period of time.

\section{F. Blink}

The number of blinks during thermal stimulus and no thermal stimulus are applied to user is measured. Fig. 9(a) shows the time series of the number of blinks data while Fig. 9(b) shows the result from linear regressive analysis between the number of blinks and thermal stimulus. Also, Table II shows the number of blinks for thermal stimulus and no thermal stimulus period of time.

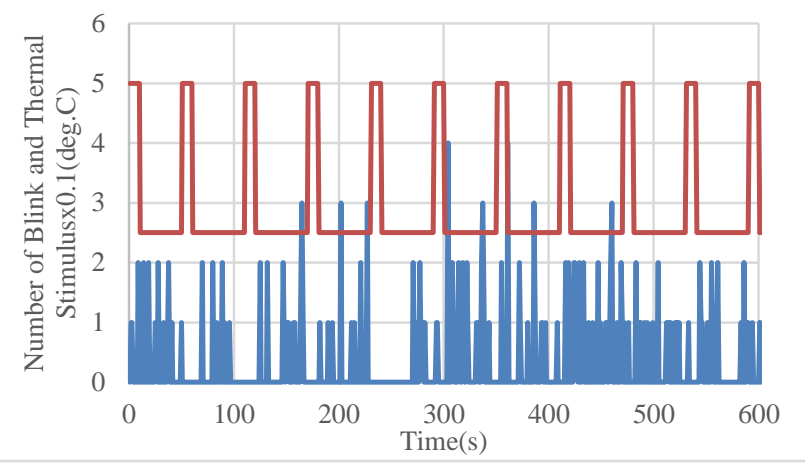

(a) Time series of data

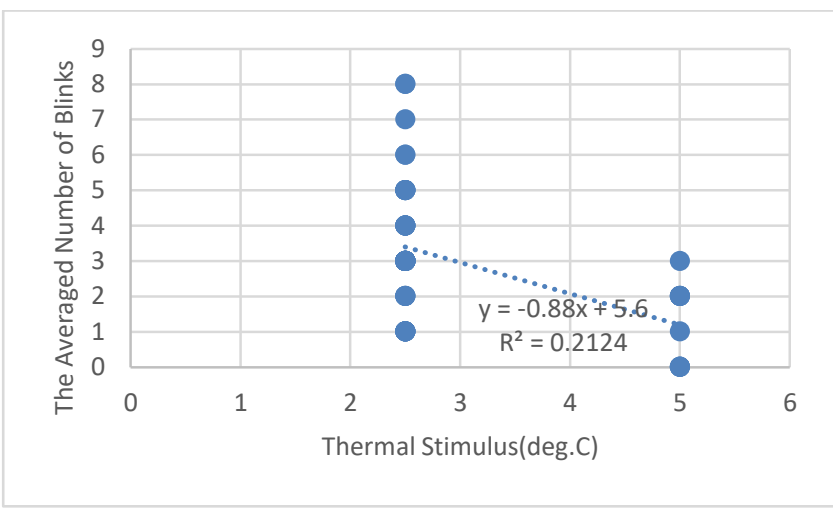

(b) Linear regression

Fig. 9. Relation between thermal stimulus and the number of blinks.

As shown in Fig. 9(b), the relation between the number of blinks and thermal stimulus is not high (correlation coefficient is around 0.113). Therefore, the number of blinks is not changed so much for thermal stimulus and no thermal stimulus period of time.

\section{G. Pupil Size Change Rate}

Meanwhile, pupil size change rate is investigated with the pupil size measured data. Human eye pupil size maybe rapidly changes due to thermal stimulus while pupil size is changed slowly for no thermal stimulus. Fig. 10(a) shows time series of pupil size change rate data while Fig. 10(b) shows the result from linear regression between thermal stimulus and the pupil size change rate. The correlation coefficient between both is approximately 0.678 . Therefore, the pupil size change rate can be a good indicator of the thermal pain.
TABLE II. THE NUMBER OF BLINKS

\begin{tabular}{|c|c|}
\hline Time Duration(s) & The Number of Blink/min. \\
\hline $1 \sim 60$ & 35 \\
\hline $61 \sim 120$ & 16 \\
\hline $121 \sim 180$ & 17 \\
\hline $181 \sim 240$ & 6 \\
\hline $241 \sim 300$ & 6 \\
\hline $301 \sim 360$ & 8 \\
\hline $361 \sim 420$ & 4 \\
\hline $421 \sim 480$ & 2 \\
\hline $481 \sim 540$ & 5 \\
\hline $541 \sim 600$ & 7 \\
\hline
\end{tabular}

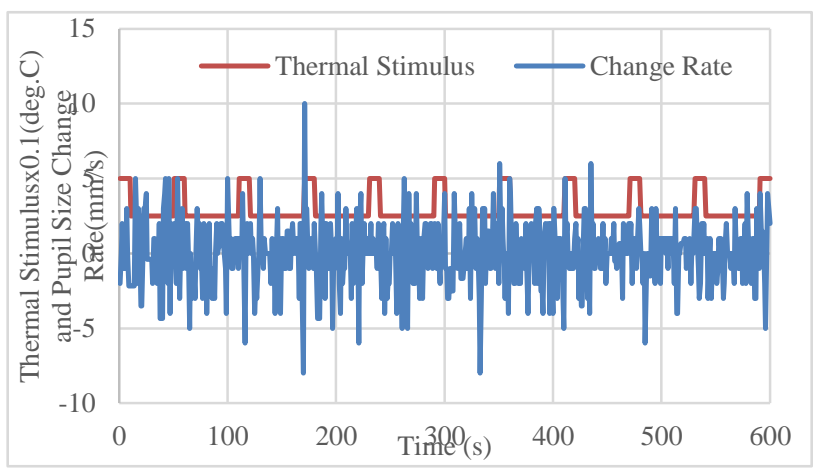

(a) Time series of data

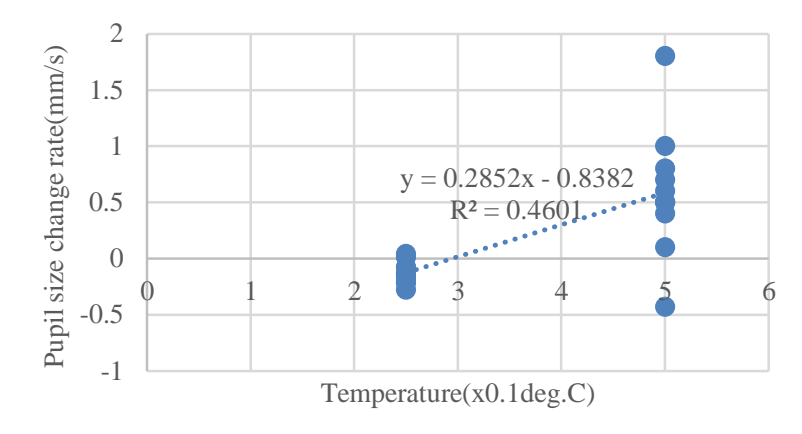

(b) Linear regression

Fig. 10. Relation between thermal stimulus and pupil size change rate.

\section{H. Pupil Center Location and Its Change Rate}

It is used to be occurred that pupil center location is changed when users feel some strange actions including thermal stimulus. Therefore, it may be possible to use these two features, pupil center location change and its change rate for detection of thermal pain. Fig. 11 shows time series of the features, pupil center location change and its change rate. In the figure, short and long radius of the ellipsoid of pupil shape, pupil center location of $\mathrm{x}$ and $\mathrm{y}$ coordinates, as well as short radius with 0 data and the difference between the short radius and short radius with 0 data are shown. 


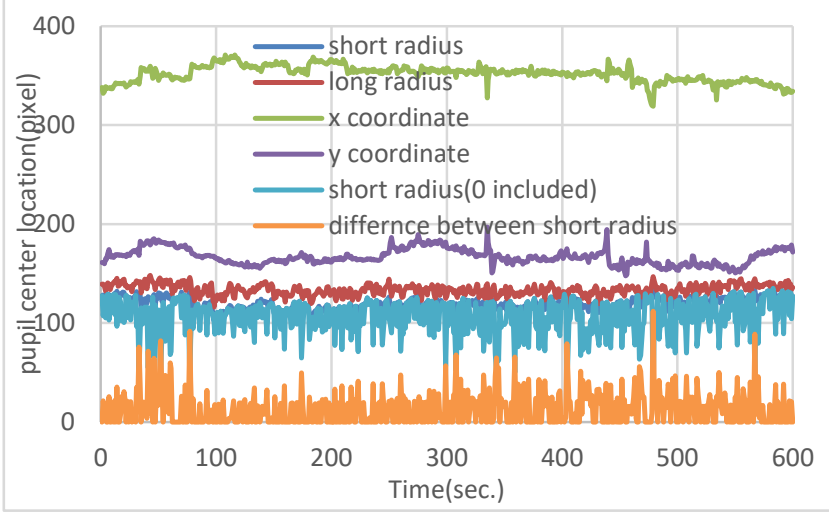

Fig. 11. Time series of features of short and long radius of the ellipsoid of pupil shape, pupil center location of $\mathrm{x}$ and $\mathrm{y}$ coordinates, as well as short radius with 0 data and the difference between the short radius and short radius with 0 data.

From the features, square of the pupil center location changes in unit of pixel and its change rate are calculated. Fig. 12(a) shows time series of pupil center location changes and thermal stimulus and Fig. 12(b) also shows the relation between thermal stimulus and the pupil center location changes.

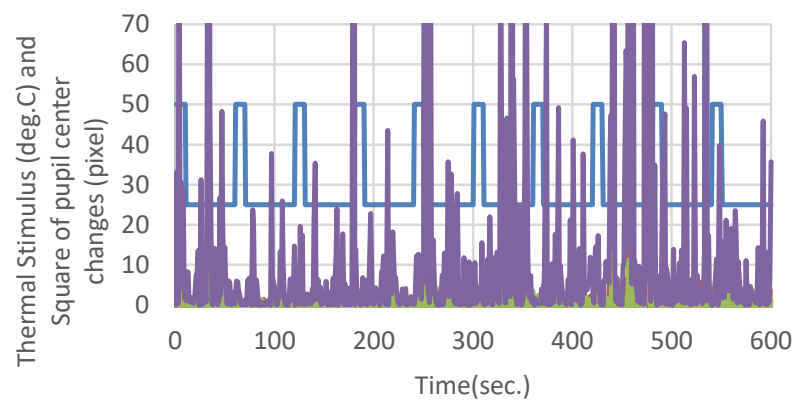

(a) Time series

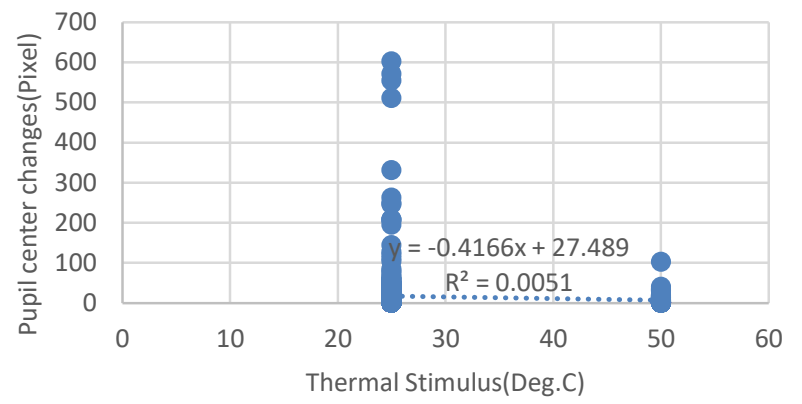

(b) Relation

Fig. 12. Time series of the pupil center location changes and thermal stimulus as well as its relation between both.

Correlation between both is quite low, 0.0051. Therefore, it is difficult to get a good classification performance with SVM. On the other hand, correlation coefficient between pupil center location change rate in unit of pixel/sec. and thermal stimulus is around 0.5 as shown in Fig. 13. Therefore, it is better than the feature of pupil center location changes and may be possible to get a good classification performance.

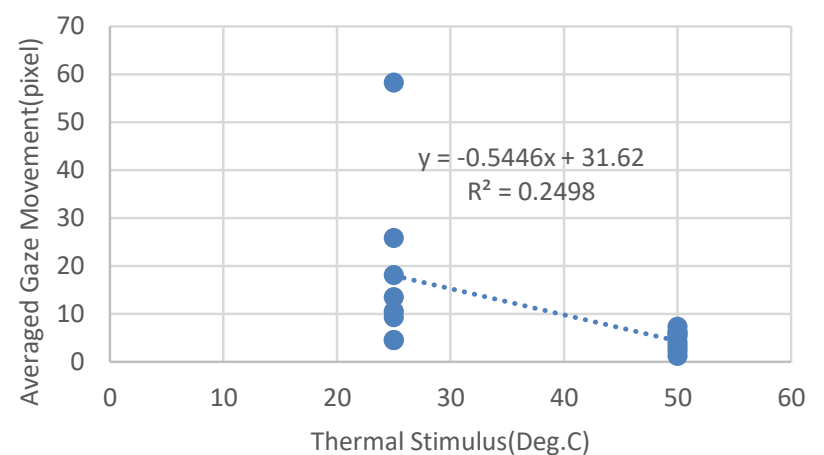

Fig. 13. Relation between the pupil center location change rate and thermal stimulus.

In summary, although the conventional method uses the feature of pupil size, pupil size change rate is much better than pupil size for the reason that correlation coefficients between thermal stimulus and the above two features are quite different.

\section{Classification Performance}

SVM based classification performances with the aforementioned four features, pupil size $(\mathrm{P})$, the number of blinks (B), eye size (E) and pupil size change rate (S) and their possible combinations are evaluated. Table III(a) shows classification performance with four single features while Table III(b) shows that with six two feature combination. Meanwhile, Table III(c) shows the classification performance with five possible three feature combinations.

TABLE III. SVM BASEd ClASSIFICATION PERFORMANCE (PAIN AND NoPAIN)

(a) Single feature

\begin{tabular}{|c|c|c|c|}
\hline $\mathbf{P}$ & $\mathbf{B}$ & $\mathbf{E}$ & $\mathbf{S}$ \\
\hline $60.97 \%$ & $75.82 \%$ & $51.3 \%$ & $62.46 \%$ \\
\hline
\end{tabular}

(b) Two feature combination

\begin{tabular}{|c|c|c|c|c|c|}
\hline $\mathbf{P}+\mathbf{B}$ & $\mathbf{P + E}$ & $\mathbf{P}+\mathbf{S}$ & $\mathbf{B}+\mathbf{E}$ & $\mathbf{B}+\mathbf{S}$ & $\mathbf{E}+\mathbf{S}$ \\
\hline $73.71 \%$ & $57.92 \%$ & $59.34 \%$ & $70.88 \%$ & $73.82 \%$ & $50 \%$ \\
\hline
\end{tabular}

(c) Three feature combination

\begin{tabular}{|l|l|l|l|l|}
\hline $\mathbf{P + B}+\mathbf{E}$ & $\mathbf{P + B}+\mathbf{S}$ & $\mathbf{P}+\mathbf{E}+\mathbf{S}$ & $\mathbf{B}+\mathbf{E}+\mathbf{S}$ & $\mathbf{P}+\mathbf{B}+\mathbf{E}+\mathbf{S}$ \\
\hline $69.86 \%$ & $74.02 \%$ & $56.94 \%$ & $69.95 \%$ & $67.48 \%$ \\
\hline
\end{tabular}

It is concluded that the number of blinks shows the best classification performance followed by the three feature combination among pupil size, the number of blinks and pupil size change rate.

\section{CONCLUSION}

Method for thermal pain level prediction with human eye motion is proposed. Through experiments, it is found that thermal pain level is much sensitive to the change rate of pupil size rather than pupil size itself. Moreover, the number of blinks shows better classification performance than the other features. 
Also, it is found that the number of blinks and the eye size are not good indicator for thermal pain. Furthermore, it is also found that user respond to the thermal stimulus so quickly ( 0 to 3 sec.) while the thermal pain is remaining for a while (4 to 17 sec.) after the thermal stimulus is removed.

\section{FUTURE STUDY}

Further study is required for more experimental data with a variety of examiners. In order to validate the proposed method, more experiments with a variety of examiners are highly required.

\section{ACKNOWLEDGMENT}

[4]

[5] Kohei Arai, Ronny Mardiaynto, Method for psychological status estimation by gaze location monitoring using eye-based HumanComputer Interaction, International Journal of Advanced Computer Science and Applications, 4, 3, 199-206, 2013.

[6] Kohei Arai, Kiyoshi Hasegawa, Method for psychological status monitoring with line of sight vector changes (Human eyes movements) detected with wearing glass, International Journal of Advanced Research in Artificial Intelligence, 2, 6, 65-70, 2013.

[7] Kohei Arai, Frequent physical health monitoring as vital sign with psychological status monitoring for search and rescue of handicapped, disabled and elderly persons, International Journal of Advanced Research in Artificial Intelligence, 2, 11, 25-31, 2013.

[8] Kohei Arai, Psychological status monitoring with cerebral blood flow: $\mathrm{CBF}$, Electroencephalogram: EEG and Electro-Oculogram: EOG measurements, International Journal of Advanced Research on Artificial Intelligence, 4, 6, 8-15, 2015.

[9] Kohei Arai, Relations between psychological status and eye movements, International Journal of Advanced Research on Artificial Intelligence, 4, 6, 16-22, 2015.

[10] Kohei Arai, Psychological status monitoring with cerebral blood flow, electroencephalogram and electrooculogram measurements, Journal of Information Technology and Computer Science, 7, 8, 1-7, 2015

[11] Kohei Arai, Rescue system with sensor network for physical and psychological health monitoring, Proceedings of the International Seminar on Intelligent Technology and Its Applications: ISITIA 2015, (Keynote Speech), 2015.
The author would like to tank Mr. Takuya Kumasaki of Saga University for his effort to conduct the experiment.

\section{REFERENCES}

[1] James C. Eisenach, Regina Curry, Carol A. Aschenbrenner, Robert C. Coghill Timothy, T.Houle, Pupil responses and pain ratings to heat stimuli: Reliability and effects of expectations and a conditioning pain stimulus, Journal of Neuroscience Method, 279, 1, 52-59, 2017.

[2] Justin E. Brown, Neil Chatterjee, Jarred Younger, Sean Mackey, Towards a Physiology-Based Measure of Pain: Patterns of Human Brain Activity Distinguish Painful from Non-Painful Thermal Stimulation, PLoS ONE 6(9): e24124, 2011.

[3] J. Kaprzy Edit., Kohei Arai, Rescue System for Elderly and Disabled Persons Using Wearable Physical and Psychological Monitoring System, Studies in Computer Intelligence, 542, 45-64, Springer $\begin{array}{llll}\text { Publishing } & \text { Co. } & \text { Ltd., } & \end{array}$

[12] D. Alnaes, M.H. Sneve, T. Espeseth, T. Endestad, S.H. van de Pavert, B. Laeng, Pupil size signals mental effort deployed during multiple object tracking and predicts brain activity in the dorsal attention network and the locus coeruleus, Journal of Visual., 14, 1-20, 2014.

\section{AUTHORS' PROFILE}

Kohei Arai, He received BS, MS and PhD degrees in 1972, 1974 and 1982, respectively. He was with The Institute for Industrial Science and Technology of the University of Tokyo from April 1974 to December 1978 also was with National Space Development Agency of Japan from January, 1979 to March, 1990. During from 1985 to 1987, he was with Canada Centre for Remote Sensing as a Post Doctoral Fellow of National Science and Engineering Research Council of Canada. He moved to Saga University as a Professor in Department of Information Science on April 1990. He was a councilor for the Aeronautics and Space related to the Technology Committee of the Ministry of Science and Technology during from 1998 to 2000. He was a councilor of Saga University for 2002 and 2003. He also was an executive councilor for the Remote Sensing Society of Japan for 2003 to 2005. He is an Adjunct Professor of University of Arizona, USA since 1998. He also is Vice Chairman of the Science Commission "A" of ICSU/COSPAR since 2008 then he is now award committee member of ICSU/COSPAR. He wrote 37 books and published 570 journal papers. He received 30 of awards including ICSU/COSPAR Vikram Sarabhai Medal in 2016, and Science award of Ministry of Mister of Education of Japan in 2015. He is now Editor-in-Chief of IJACSA and IJISA. http://teagis.ip.is.saga-u.ac.jp/index.ht 